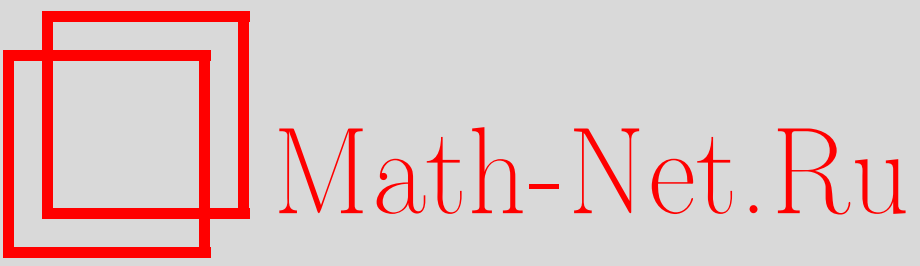

А. А. Коньков, Теоремы сравнения для дивергентных эллиптических неравенств, содержащих члены с младшими производными, УМН, 2004, том 59, выпуск $5,151-152$

DOI: https://doi.org/10.4213/rm779

Использование Общероссийского математического портала Math-Net.Ru подразумевает, что вы прочитали и согласны с пользовательским соглашением

http: //www . mathnet.ru/rus/agreement

Параметры загрузки:

IP: 18.234 .197 .8

26 апреля 2023 г., 18:15:15 


\title{
ТЕОРЕМЫ СРАВНЕНИЯ ДЛЯ ДИВЕРГЕНТНЫХ ЭЛЛИПТИЧЕСКИХ НЕРАВЕНСТВ, СОДЕРЖАЩИХ ЧЛЕНЫ С МЛАДШИМИ ПРОИЗВОДНЫМИ
}

\author{
A. А. Коньков
}

Пусть $\Omega$ - открытое подмножество $\mathbb{R}^{n}, n \geqslant 2$, а $L$ - дифференциальный оператор вида

$$
L=\sum_{i, j=1}^{n} \frac{\partial}{\partial x_{i}}\left(a_{i j}(x) \frac{\partial}{\partial x_{j}}\right)+\sum_{i=1}^{n} b_{i}(x) \frac{\partial}{\partial x_{i}}
$$

с локально ограниченными измеримыми коэффициентами, удовлетворяющими условию равномерной эллиптичности

$$
C_{1}|\xi|^{2} \leqslant \sum_{i, j=1}^{n} a_{i j}(x) \xi_{i} \xi_{j} \leqslant C_{2}|\xi|^{2}, \quad C_{1}, C_{2}>0
$$

для всех $x=\left(x_{1}, \ldots, x_{n}\right) \in \Omega, \xi=\left(\xi_{1}, \ldots, \xi_{n}\right) \in \mathbb{R}^{n}$.

Договоримся о следуюших обозначениях: $Q_{r}=\{x:|x|<r\}, B_{r}=\{x:|x| \leqslant r\}$ и $S_{r}=\{x$ : $|x|=r\}$. По умолчанию будем предполагать, что $0 \leqslant R_{0}<R \leqslant \infty$ и при этом $\Omega \cap S_{r} \neq \varnothing$ для любого $r \in\left(R_{0}, R\right)$.

Неотрицателная функция $u \in W_{2, \text { loc }}^{1}\left(\Omega \cap Q_{R} \backslash B_{R_{0}}\right) \cap L_{\infty, \text { lос }}\left(\Omega \cap Q_{R} \backslash B_{R_{0}}\right)$ называется решением неравенства

$$
L u \geqslant F(x, u) \text { в } \Omega \cap Q_{R} \backslash B_{R_{0}},
$$

где $F: \Omega \times[0, \infty) \rightarrow[0, \infty)$, если $F(\cdot, u(\cdot)) \in L_{\infty, \text { lос }}\left(\Omega \cap Q_{R} \backslash B_{R_{0}}\right)$ и при этом

$$
\begin{aligned}
& -\int_{\Omega \cap Q_{R} \backslash B_{R_{0}}} \sum_{i, j=1}^{n} a_{i j}(x) \frac{\partial u}{\partial x_{j}} \frac{\partial \varphi}{\partial x_{i}} d x \\
& \quad+\int_{\Omega \cap Q_{R} \backslash B_{R_{0}}} \sum_{i=1}^{n} b_{i}(x) \frac{\partial u}{\partial x_{i}} \varphi d x \geqslant \int_{\Omega \cap Q_{R} \backslash B_{R_{0}}} F(x, u) \varphi d x
\end{aligned}
$$

для любой неотрицательной функции $\varphi \in C_{0}^{\infty}\left(\Omega \cap Q_{R} \backslash B_{R_{0}}\right)[1]$.

Пишем также, что

$$
\left.u\right|_{\partial \Omega \cap Q_{R} \backslash B_{R_{0}}}=0,
$$

если $\eta u \in \stackrel{\circ}{W} \frac{1}{2}(\Omega)$ для любой функции $\eta \in C_{0}^{\infty}\left(Q_{R} \backslash B_{R_{0}}\right)$. В случае $\partial \Omega \cap Q_{R} \backslash B_{R_{0}}=\varnothing$ условие (2), очевидно, справедливо для всякой функции $u \in W_{2, \mathrm{loc}}^{1}\left(\Omega \cap Q_{R} \backslash B_{R_{0}}\right)$.

Обозначим, далее,

$$
M(r ; u)=\underset{S_{r} \cap \Omega}{\operatorname{ess} \sup } u .
$$

Вопросы, рассматриваемые в этом сообщении, исследовались рядом авторов [1]-[4]. Ниже мы приводим две теоремш сравнения для дивергентных эллиптических неравенств вида (1). В недивергентном случае аналогичные результаты были ранее доказаны автором в работе [2].

Работа выполнена при поддержке гранта Президента РФ "Ведущие научные школы" № 1464. 2003.1. 
Tеорема 1. Пусть $\sigma>1, k_{1}>0, k_{2}>0$ - некоторье вещественные числа, и пусть $b:\left[R_{0}, \infty\right) \rightarrow[0, \infty)$ и $f:\left[R_{0}, \infty\right) \times(0, \infty) \rightarrow[0, \infty)$ - локально ограниченные измеримые функции такие, что

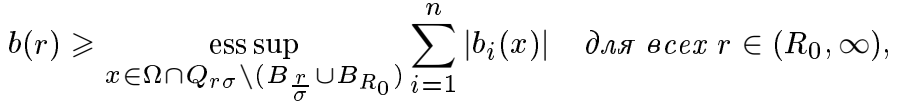

$$
\begin{aligned}
& f(r, t) \leqslant \underset{x \in \Omega \cap Q_{r \sigma} \backslash\left(B_{\frac{r}{\sigma}} \cup B_{R_{0}}\right)}{\operatorname{essinf}} F(x, t) \quad \text { для всех } r \in\left(R_{0}, \infty\right), t \in(0, \infty) \text {, } \\
& f(r, t-0)=f(r, t) \quad \text { для всех } r \in\left(R_{0}, \infty\right), t \in(0, \infty), \\
& f\left(r, t_{1}\right) \leqslant f\left(r, t_{2}\right) \quad \text { для всех } r \in\left(R_{0}, \infty\right), 0<t_{1} \leqslant t_{2} .
\end{aligned}
$$

Предположим, что и - неотрицательное решение задачи (1), (2), удовлетворяющее условиям

$$
\begin{gathered}
M\left(R_{0}+0 ; u\right)>0, \\
M\left(r_{1} ; u\right) \leqslant M\left(r_{2} ; u\right) \quad \text { для всех } R_{0}<r_{1} \leqslant r_{2}<R .
\end{gathered}
$$

Тогда найдутся константы $\alpha>0, \beta>0$, зависящие только от $n, \sigma, k_{1}, k_{2}$, а также от постоянных әллиптичности $C_{1}, C_{2}$ оператора $L$, такие, что на промехутке $\left[R_{0}, R\right)$ существует решение задачи Коши

$$
\begin{gathered}
m^{\prime \prime}+\left(\frac{1+k_{1}}{r}+k_{2} b(r)\right) m^{\prime}=\alpha f(r, \beta m), \\
m\left(R_{0}\right)=M\left(R_{0}+0 ; u\right), \quad m^{\prime}\left(R_{0}\right)=0,
\end{gathered}
$$

для которого при всех $r \in\left(R_{0}, R\right)$ справедлива оченка

$$
M(r ; u) \geqslant m(r)>0 .
$$

ЗАмечАниЕ 1 . В случае $n \geqslant 3$, полагая $k_{1}=n-2$ и $k_{2}=1$, получим в левой части уравнения (3) радиальную компоненту оператора $\Delta+b(|x|) \nabla|x| \cdot \nabla$.

Теорема 1 вытекает из следующего утверждения.

Теорема 2. Пусть выполнены предположения теоремы 1. Тогда найдутся константы $\alpha>0, \beta>0$, зависящие только от $n, \sigma, k_{1}, k_{2}, C_{1}, C_{2}$, такие, что для любого $r \in\left(R_{0}, R\right)$

$2 \partial e$

$$
M(r ; u)-M\left(R_{0}+0 ; u\right) \geqslant \alpha \int_{R_{0}}^{r} K(r, \xi) f(\xi, \beta M(\xi ; u)) d \xi
$$

$$
K(r, \xi)=\int_{\xi}^{r} d \rho\left(\frac{\xi}{\rho}\right)^{1+k_{1}} e^{-k_{2} \int_{\xi}^{\rho} b(s) d s} .
$$

\section{СПИСОК ЛИТЕРАТУРЫ}

[1] О.А. Ладыженская, Н.Н. Уральцева. Линейные и квазилинейные уравнения эллиптического типа. М.: Наука, 1964. [2] А. А. Kon'kov // Nonlinear Anal. 2000. V. 42. №7. Р. 1253-1270. [3] В. А. Кондратьев, Е. М. Ландис // Матем. сб. 1988. Т. 135. №3. С. 346-360. [4] Э. Митидиери, С. И. Похожаев // Тр. МИАН. 2001. Т. 234. С. 1-384.

Московский государственньй Принято редколлегией университет им. М. В. Ломоносова 23.07.2004 